\title{
CONSTRUCCIÓN DE UN DICCIONARIO DE COMPORTAMIENTOS Y COMPETENCIAS GENÉRICAS INDISPENSABLES PARA EL SACERDOTE DIOCESANO EN PERÚ
}

\section{Construction of a dictionary of behaviors and generic skills essential for the diocesan priest in Peru}

\author{
Jean-Paul Swinnen Sardón*
}

\begin{abstract}
Resumen
La investigación que se reporta parte de un estudio previo en el que se construyó un perfil de competencias genéricas para el sacerdote diocesano en Perú. Es de carácter exploratorio $y$ descriptivo. Sigue un paradigma de investigación cualitativa. Busca establecer los comportamientos necesarios para la realización de la labor del sacerdote diocesano en Perú. Para ello realiza dos estudios. En el primero identifica cinco competencias genéricas indispensables: Vida interior, Obediencia, Liderar con el ejemplo, Fraternidad sacerdotal y Formación permanente. En el segundo, construye y valida un diccionario de comportamientos para evaluar el nivel de apropiación de cada competencia genérica indispensable. Proporciona indicadores concretos y mensurables del desempeño estándar del sacerdote diocesano en Perú.
\end{abstract}

Palabras clave: Diccionario de competencias, Competencias genéricas, Validación de contenido, Competencias del sacerdote diocesano

\begin{abstract}
The present article is based on a previous study that provided a competencies-based profile of a Peruvian diocesan priest. It is of an exploratory and descriptive nature, and follows the qualitative research methodology. It seeks to establish the behaviors that are necessary for carrying out the pastoral work of a Peruvian diocesan priest. For that purpose, it carries out two studies. In the first one, it identifies five essential generic competencies: Interior Life, Obedience, Leading by Example, Priestly Fraternity and Continuous Education. In the second one, it creates and validates a behavioral dictionary for assessing levels of assimilation of each of the essential generic competencies. Moreover, it provides concrete and measurable indicators of the standard performance of a Peruvian diocesan priest.
\end{abstract}

Keywords: Dictionary of competencies, Generic competencies, Content validation, Competencies of the diocesan priest

\footnotetext{
* Psicólogo con práctica clínica privada en la ciudad de Lima.jpswinnen@gmail.com
} 
1. EL SACERDOTE DIOCESANO EN LA ORGANIZACIÓN DE LA IGLESIA CATÓLICA

La Iglesia Católica es una institución fundada por Jesucristo con el fin de servir a la humanidad (Concilio ecuménico Vaticano II, 1964, Constitución dogmática Lumen gentium, núm. 3). Todos los católicos bautizados forman parte de ella. Para cumplir con su misión institucional, la Iglesia Católica tiene una organización jerárquica. Es gobernada por los obispos, presididos por el obispo de Roma.

Desde un punto de vista territorial, la Iglesia Católica se organiza en circunscripciones geográficas, denominadas jurisdicciones eclesiásticas o diócesis, gobernadas por un obispo diocesano, quien a su vez puede tener asignados, como apoyo en su servicio pastoral, a uno o más obispos auxiliares.

Los obispos tienen a su cargo la organización del servicio pastoral a través de la actividad de las parroquias, las cuales ocupan un espacio geográfico determinado. Las parroquias están a cargo de presbíteros, es decir, sacerdotes, los cuales tienen como misión, la atención pastoral y el anuncio del mensaje de Jesucristo (Catecismo de la Iglesia Católica, 1992).

Los sacerdotes que dependen directamente de una diócesis, y no forman parte de un instituto de vida consagrada en la Iglesia Católica, son denominados, sacerdotes diocesanos.

La persona que siente el llamado vocacional al sacerdocio, y que se quiere desempeñar como sacerdote en el ámbito diocesano, tiene que realizar un proceso de discernimiento y formación integral, el cual tiene como primer requisito el ser admitido en un seminario, por el obispo de la jurisdicción eclesiástica correspondiente (Código de Derecho Canónico [CIC], can. $241 \S 1$ ). Allí inicia un proceso de formación, cuyo objetivo es consolidar el discernimiento vocacional del candidato y prepararlo para que alcance la idoneidad requerida, para el ejercicio de su labor ministerial (CIC, can. 235 § 1). El proceso de formación, se realiza ordinariamente en un seminario diocesano (CIC, can. 235 §§ 1-2).

El candidato al sacerdocio requiere realizar estudios superiores en filosofía y teología. Además, es alentado a cultivarse en los distintos aspectos humanos y espirituales propios del servicio que va a realizar (CIC, can. 244).

Finalizado el proceso de formación, el candidato queda listo para ser consagrado como diácono y luego como sacerdote. El diaconado es la etapa previa al presbiterado, que da inicio propiamente al servicio ministerial del sacerdote.

La Congregación para la Educación Católica (2008), que tiene entre sus responsabilidades la formación sacerdotal, sostiene que la labor del sacerdote, requiere de cualidades humanas, además de virtudes morales y teologales que deben estar respaldadas por el equilibrio humano y psíquico del candidato. Enumera un amplio elenco de cualidades, entre las que destacan, la necesidad del cultivo de habilidades sociales, un claro sentido de pertenencia, que luego posibilite el vínculo de trabajo con el obispo, capacidad de entusiasmarse con ideales e integridad para vivirlos, capacidad de toma de decisiones, fidelidad al compromiso adquirido, conciencia de los propios recursos y limitaciones, capacidad de integrar críticas, y capacidad de confiar y de acoger al prójimo.

\section{COMPETENCIAS GENÉRICAS INDISPENSABLES PARA EL SACERDOTE DIOCESANO EN PERÚ}

En este artículo, se reporta una investigación en la que se buscó establecer los comportamientos necesarios para el desempeño estándar del sacerdote diocesano en su labor, desde un enfoque por competencias genéricas.

Las competencias, son definidas como "aquellos comportamientos observables y habituales que posibilitan el éxito de una persona en una actividad o función" (Tobón, 2009, p. 42). La identificación de competencias, tiene como objetivo, encontrar un estándar evaluable de rendimiento y una guía para lograrlo (Kahane, 2008).

Un enfoque por competencias genéricas, permite identificar habilidades laborales que, si bien toman características propias según el tipo de ocupación, son reconocibles y válidas en muy diversos ambientes, independientemente de factores tales como, tipo de empleo, o de industria, o regiones geográficas (Saracho, 2005). 
Según Saracho (2005), las competencias son definidas como genéricas, cuando el enfoque metodológico acentúa los comportamientos requeridos para un desempeño estándar en un cargo. Este enfoque se observa en los estudios de Torres y Gutiérrez (2007), Oliva y Henson (1980), Alonso-Martín (2010), Nikolaou (2003), Finney (2009) y Gvaramadze (2012), entre otros.

Los comportamientos, son indicadores que permiten evaluar el nivel de apropiación de la competencia en el individuo (Alles, 2009). Dado que las responsabilidades y labores de los sacerdotes diocesanos varían notablemente según el ámbito del servicio que les confíe el obispo, para un estudio de sus comportamientos resulta útil el enfoque por competencias genéricas definido por Saracho (2005). Éste, permite centrarse en las características comunes de la labor sacerdotal, en cualquiera de sus múltiples escenarios, y proponer los comportamientos requeridos para el desempeño estándar del sacerdote diocesano en Perú.

Para evaluar el cumplimento de los requisitos estándar de cada competencia genérica es necesario construir un diccionario de comportamientos. Alles (2009) considera que un diccionario de comportamientos facilita reconocer el nivel de apropiación de cada competencia, describiendo los indicadores que corresponden a un nivel insuficiente, estándar, superior o muy superior.

En esta investigación, se partió de las competencias genéricas del sacerdote diocesano en Perú, definidas en un estudio previo (Swinnen, 2014). Dicho estudio, propuso dieciséis competencias genéricas, identificadas luego de un análisis de contenido de quince entrevistas a obispos en Perú, en cuanto poseedores de una experiencia sacerdotal institucionalmente reconocida. Teniendo como base las competencias génericas propuestas en dicho estudio, en esta investigación se ha querido identificar las indispensables y construir con ellas un diccionario de comportamientos para el ejercicio de la labor del sacerdote diocesano en Perú.

Esta investigación, es de carácter exploratorio, dada la ausencia de literatura en torno a perfiles por competencias de sacerdotes diocesanos. No se ha encontrado otros estudios que definan un perfil sacerdotal diocesano en Perú o en otros territorios. Es, asimismo, de carácter descriptivo, en cuanto que busca desarrollar los comportamientos identificados a partir de la experiencia de los obispos entrevistados (Cortada de Kohan, Macbeth \& López, 2008). El paradigma de investigación asumido, es el cualitativo (Vasilachis de Gialdino, 2006).

La pregunta de investigación fue: “¿Cuáles son los comportamientos necesarios para la realización de la labor del sacerdote diocesano en Perú?"

Esta pregunta fue dividida a su vez en otras dos: la primera, “¿Cuáles son las competencias genéricas indispensables del sacerdote diocesano en Perú?", y la segunda, "¿Qué comportamientos evidencian los niveles de apropiación de las competencias genéricas indispensables del sacerdote diocesano en Perú?". Para responder a ellas se realizaron dos estudios.

\section{IDENTIFICACIÓN DE LAS COMPETENCIAS GENÉRICAS INDISPENSABLES DEL SACERDOTE DIOCESANO EN PERÚ}

El primer estudio, tuvo como objetivo, determinar qué competencias genéricas, son consideradas indispensables por la instancia más representativa de la organización, en este caso, de la Iglesia Católica en Perú.

\subsection{Participantes}

Siguiendo con la metodología planteada por Saracho (2005), se buscó la instancia de mayor representatividad institucional. Se reconoció para este papel al obispo presidente de la Conferencia Episcopal Peruana, elegido para tal cargo por los demás obispos peruanos, quien accedió a participar en la investigación.

Este tipo de muestra es no probabilístico e intencional, donde la elección de los sujetos, "no depende de que todos los sujetos tengan la misma probabilidad de ser elegidos, sino de la decisión del investigador" (Hernández, Fernández \& Baptista, 2006, p. 84). 


\subsection{Método}

Seexplicó, alainstanciade mayor representatividad institucional, el sentido de la investigación y las características de su participación. A continuación, se le preguntó si estaba de acuerdo con los términos indicados, aceptando y firmando un consentimiento informado. Se le solicitó que, en cuanto, representante de los obispos de Perú, analizase las dieciséis competencias genéricas construidas en la investigación "Construcción de un perfil por competencias genéricas para el sacerdote diocesano en Perú" (Swinnen, 2014), y discriminase, cuáles consideraría indispensables, para la formación del sacerdote diocesano en Perú. Luego de revisar las dieciséis competencias, determinó cinco de ellas como indispensables debido a su relevancia y a su representatividad del quehacer del sacerdote peruano.

\subsection{Resultados}

Las cinco competencias genéricas identificadas como indispensables para el ejercicio del sacerdocio diocesano en Perú, fueron: Vida interior, Obediencia, Liderar con el ejemplo, Fraternidad sacerdotal y Formación permanente, definidas del siguiente modo en el estudio base:

1. Vida interior. Inquietud por cultivar diariamente una relación personal con Cristo a través de la experiencia cotidiana, la oración, el recurso a los sacramentos, y el esfuerzo por conocer a Cristo, cada vez más.

2. Obediencia. Disposición para aceptar, asumiendo como decisiones propias, las de quien ejerce la autoridad, sin que éstas se opongan a la justicia o moralidad. Esto implica, una disposición a realizar con prontitud y adhesión lo decidido.

3. Liderar con el ejemplo. Capacidad para promover y comunicar los ideales, y los valores de la fe católica, a través de un modelo de conducta personal, coherente con los consejos evangélicos (la obediencia religiosa, el celibato y la pobreza) para buscar seguir a Cristo con la propia vida, así como la adhesión a las enseñanzas del magisterio de la Iglesia.

4. Fraternidad sacerdotal. Capacidad para buscar, establecer y cultivar relaciones significativas y duraderas fundadas en la caridad cristiana, el compañerismo y la solidaridad con otros sacerdotes/ seminaristas. Implica también el trabajar de manera cooperativa y activa entre los mismos.

5. Formación permanente. Disposición permanente a velar por el estudio en áreas relacionadas al servicio pastoral; cultivo de áreas de interés personal/profesional; conocimiento y comprensión del magisterio de la Iglesia Católica, con miras a su propio desarrollo personal y aporte pastoral.

El representante de los obispos de Perú, reconoció que estas competencias genéricas, son las que, a su criterio, representan con mayor relevancia el quehacer del sacerdote diocesano en Perú.

Elestudio,nodemandóquesehicieradistincionesde mayor o menor importancia al momento de seleccionar las competencias. No obstante, el entrevistado manifestó un orden de relevancia más representativo a su juicio. El elenco reportado ha conservado dicho orden, de mayor a menor relevancia.

\section{DICCIONARIO DE COMPORTAMIENTOS DE LAS COMPETENCIAS GENÉRICAS INDISPENSABLES DEL SACERDOTE DIOCESANO EN PERÚ}

El segundo estudio tuvo como objetivo construir un diccionario de comportamientos de las competencias genéricas indispensables del sacerdote diocesano en Perú.

\subsection{Participantes}

Para este estudio se recurrió a las entrevistas realizadas en el estudio base (Swinnen, 2014), a partir de las cuales se había construido el perfil de dieciséis competencias genéricas. El diseño de la muestra en dicho estudio fue también no probabilístico e intencional. Tomándose a partir de una población de cincuenta y dos obispos en Perú (Cheney, 2013). La muestra, estuvo sujeta a la disponibilidad de los mismos. Se entrevistó a quince de dichos obispos, punto en el que se alcanzó la saturación teórica (Strauss \&Corbin, 1991). Los obispos participantes, procedieron de distintas ciudades de Perú. Las visitas fueron realizadas a lo largo de tres meses. El criterio de inclusión, fue 
que los obispos ejerzan o hayan ejercido su servicio, en alguna diócesis de Perú, por un período mayor a un año. Se consideró irrelevante, que en el momento de la entrevista, estuviesen ejerciendo su labor pastoral o estuviesen retirados. La media de edad fue de sesenta y seis años. Tres ejercían como obispos auxiliares, seis eran obispos titulares y seis eméritos (que ya no ejercen su función por razones de edad), diez provenían de órdenes y congregaciones religiosas, y habían sido asimilados al clero diocesano, desde el momento en el que fueron nombrados obispos; cinco siempre habían sido sacerdotes diocesanos que se habían formado en seminarios diocesanos, siendo finalmente ordenados obispos. Uno era de nacionalidad italiana, cuatro de nacionalidad española, ocho oriundos de Lima y dos oriundos de otras provincias de Perú. Los quince fueron educados en colegios católicos y provenían de familias que se definían católicas, todos hijos de matrimonios casados por el rito católico. Tres de los quince tenían una segunda carrera además de la teológica.

\subsection{Método}

Se revisaron las transcripciones de las entrevistas realizadas en el estudio base, seleccionando los contenidos correspondientes a las cinco competencias reconocidas como indispensables y se identificó a partir de ellas los comportamientos correspondientes a cada una de ellas. Se organizaron los comportamientos de acuerdo a los siguientes niveles de apropiación en una escala ordinal: (1) necesita mejorar, (2) estándar, (3) superior, y (4) muy superior. Con ello se elaboró una primera versión del diccionario de comportamientos, la cual fue sometida a la consulta de dos psicólogos expertos en el ámbito organizacional. Con su aporte, el diccionario fue revisado exhaustivamente, dando lugar a ocho versiones. Los expertos corroboraron la consistencia de la construcción de los cuatro niveles propuestos para cada competencia. A continuación a sometió el diccionario de comportamientos al juicio de otros ocho psicólogos, quienes validaron su contenido, con una concordancia de .93 según el coeficiente de validez V de Aiken (Escurra, 1989). La validación determinó que los comportamientos establecidos dan significativamente razón del nivel de apropiación de cada competencia en cada uno de los cuatro niveles presentes en el diccionario de comportamientos.

\subsection{Resultados}

Se reporta a continuación el diccionario de comportamientos de competencias genéricas indispensables para el sacerdote diocesano en Perú, construido luego de la revisión de las transcripciones de las entrevistas del estudio base, la identificación de los comportamientos correspondientes a cada competencia, y la validación realizada con el recurso a jueces.

\section{DICCIONARIO DE COMPORTAMIENTOS DE COMPETENCIAS GENÉRICAS INDISPENSABLES PARA EL SACERDOTE DIOCESANO EN PERÚ, SEGÚN NIVELES DE APROPIACIÓN DE CADA COMPETENCIA}

\section{Liderar con el ejemplo}

1.1 Necesita mejorar

- Evidencia en su vida privada y pública contradicciones entre sus opciones de fe y sus opciones prácticas (opuestas a los consejos evangélicos y opuestas a las enseñanzas de la Iglesia católica; p.ej.: promoción del aborto).

- Hace ostentación de seguir creencias distintas a las enseñadas por Cristo y expresadas por el magisterio de la Iglesia.

- Realiza un esfuerzo mínimo por comportarse de acuerdo a los valores que Cristo enseñó y que el magisterio de la Iglesia explica. Sólo lo hace en circunstancias específicas y cuando se lo requiere. No es parte de su vida diaria.

\subsection{Estándar}

- Esfuerzo activo: hace un esfuerzo activo para interiorizar y respetar las enseñanzas de Cristo, expresadas a través del magisterio de la Iglesia.

- Muestra compromiso con los consejos evangélicos, en cuanto ideales personales que busca plasmar en su vida cotidiana.

- Promueve el cumplimiento de los principios y valores testimoniados, y enseñados por Cristo, cada vez que se presenta la oportunidad, y con las personas con las que interactúa. 


\subsection{Superior}

- Entiende y apoya, las decisiones y lineamientos, enseñados por el magisterio de la Iglesia.

- Busca y promueve nuevos espacios donde pueda difundir los principios, valores e ideales que Cristo enseñó, más allá de los espacios en los que habitualmente realiza su actividad pastoral.

\subsection{Muy superior}

- Su estilo cotidiano de vida es modélico en lo que respecta a la vivencia de los consejos evangélicos.

- Es un punto de referencia y testimonio a quien los demás acuden en búsqueda de orientación en su seminario y/o iglesia local para llevar una vida más comprometida con Cristo.

- Toma iniciativas personales en la difusión de las enseñanzas de Cristo y del magisterio de la Iglesia en el cual busca estar actualizado.

\section{Fraternidad sacerdotal}

\subsection{Necesita mejorar}

- Sólo participa en eventos, o se comunica con otros seminaristas, cuando se lo requieren.

- No busca establecer relaciones entre los demás seminaristas.

\subsection{Estándar}

- Entiende la importancia de estar en relación con los demás seminaristas.

- Participa en los eventos de integración con compañeros de manera activa. Le agrada relacionarse con sus compañeros y pares.

- Brinda apoyo a otros seminaristas de manera desinteresada cuando se lo solicitan.

\subsection{Superior}

- Busca espacios, por iniciativa propia, para promover las relaciones fraternas entre seminaristas.

- Se preocupa por los demás compañeros y está pendiente de apoyar cuando lo necesiten.

- Se adelanta para atender las necesidades de otros seminaristas y ofrece su apoyo aun cuando no lo hayan solicitado.

- En ocasiones es capaz de anteponer las necesidades de sus compañeros a las propias.

\subsection{Muy superior}

- Promueve la integración entre los seminaristas a través de iniciativas personales.

- Suele ser referente de fraternidad por las acciones continuas y la cercanía de sus relaciones con todos los demás seminaristas.

\section{Vida interior}

\subsection{Necesita mejorar}

- Se centra en cumplir, estrictamente, la oración oficial de la Iglesia y las celebraciones litúrgicas por rutina u obligación más que por convicción.

- Demuestra interés, limitado, por establecer una propia rutina de oración o acciones que alienten la oración diaria y a cultivar una relación personal con Cristo.

- Puede promover el desgano en sus compañeros o comenta lo agotador de esas acciones o la poca comprensión en su ejecución.

\subsection{Estándar}

- Se muestra interesado en cultivar, diariamente, su relación con Cristo a través de la oración personal y de la participación en las celebraciones litúrgicas.

- Entiende la importancia de desarrollar la vida espiritual, de manera cotidiana.

- Fomenta este interés entre sus compañeros.

- Practica con interés y constancia la oración oficial de la Iglesia y la participación en las celebraciones litúrgicas.

- Tiene espacios fijos de oración personal que suele respetar.

\subsection{Superior}

- Además de la realización diaria de la oración oficial de la Iglesia participa activamente en la promoción de la Adoración al Santísimo, el rezo diario del rosario y el cultivo de piedades en su propia vida (devoción a advocaciones marianas, a diversos santos, peregrinaciones, ofrecimientos personales, oración de novenas).

- Se muestra constantemente preocupado y entusiasmado por mejorar su relación con Cristo a través de la oración y de su labor pastoral.

- Brinda y recibe retroalimentación de sus 
compañeros seminaristas sobre su vida de oración y sobre su inquietud por conocer más a Cristo (recomendarse mutuamente lecturas, oraciones, piedades).

\subsection{Muy superior}

- Promueve actividades que fomenten el crecimiento de la vida de oración, de los demás seminaristas.

- Su crecimiento espiritual, se refleja en su dedicación y celo pastorales, y en el trato caritativo hacia los seminaristas

- Suele ser referente en cuanto a cultivo de vida interior entre los demás seminaristas.

\section{Obediencia}

\subsection{Necesita mejorar}

- Cumple con las disposiciones y acentos formativos del seminario a vista de sus formadores, pero puede ignorar algunas cuando no lo están observando.

- Critica algunas normas y principios básicos de la disciplina propia de la vida en el seminario (p.ej.: la observancia de los consejos evangélicos; las políticas formativas, los contenidos de los cursos de formación). No busca aclarar sus críticas o descontento con las instancias adecuadas.

- Se suele oponer activamente a indicaciones que no vayan de acuerdo a sus preferencias o ideas.

\subsection{Estándar}

- Entiende la importancia de cumplir con la disciplina y orientaciones de la dinámica formativa en el seminario. Entiende que ésta está ligada a la obediencia que debe a Cristo a través de las autoridades que la Iglesia ha puesto a su servicio.

- Cuando algo le parece inadecuado, impreciso o negativo en las políticas formativas busca comentarlo de manera asertiva a su formador para buscar aclarar dudas.

- Cuando obedece lo hace con conciencia de que a quien obedece es a Cristo a través de sus autoridades.
4.3 Superior

- Cumple de manera comprometida con la disciplina de su régimen de vida (observancia de los consejos evangélicos, disponibilidad para responder con prontitud a las exigencias del horario, disponibilidad para salir al encuentro de los trabajos voluntarios).

- Es diligente en estarenterado de las orientaciones pastorales del obispo de su diócesis, de las orientaciones pastorales del Santo Padre y las busca integrar en su trabajo pastoral con adhesión personal.

- Promueve en los demás por convicción la comprensión y cumplimiento de las enseñanzas de la Iglesia Católica en materias de fe y moral.

- Busca estar disponible a los requeri-mientos que sus autoridades puedan necesitar o le pidan.

\subsection{Muy superior}

- Promueve actividades que permitan y faciliten el acercamiento a Cristo a través del conocimiento del Evangelio, de las orientaciones pastorales del obispo y de las enseñanzas del Santo Padre.

- Suele ser referente de obediencia y adhesión interior al formador, al obispo y a las enseñanzas del magisterio de la Iglesia.

- En el ámbito de la vida pública manifiesta su adhesión a las iniciativas del obispo y a las orientaciones del magisterio de la Iglesia, a lo cual también alienta al resto de los católicos.

\section{Formación permanente}

\subsection{Necesita mejorar}

- Le cuesta establecer una rutina de estudio, manifiesta desgano para establecer metas en su formación personal.

- No cumple con las metas y orientaciones para su desarrollo personal en el cumplimento del programa académico del seminario.

- Demuestra poca disposición a ir más allá de lo solicitado.

- Puede promover el desgano en sus compañeros. 


\subsection{Estándar}

- Demuestra interés en capacitarse en áreas de servicio pastoral, litúrgico y doctrinal así como en otras áreas de interés intelectual/ profesional.

- Sigue las orientaciones de formación otorgadas por su formador en el seminario.

- Fomenta este interés entre sus compañeros.

- Muestra claro interés frente a la información y aprendizaje para su desarrollo personal y pastoral.

\subsection{Superior}

- Comparte su conocimiento y lo aplica al ámbito pastoral, orienta a sus compañeros y se muestra interesado en atender las necesidades de desarrollo de sus pares.

- Manifiesta inquietud por buscar ampliar su conocimiento más allá del programa de estudios que se espera que cumpla.

- Busca constante retroalimentación acerca de sus inquietudes intelectuales con compañeros y profesores.

\subsection{Muy superior}

- Promueve el intercambio y el diálogo en torno a iniciativas formativas y pastorales en el seminario.

- Se interesa por mantener un conoci $\neg$ miento actualizado en temas propios de su formación teológica, y pastoral así como de otros intereses intelectuales que valora por su posible aplicación al ámbito de servicio en el que se desempeñará como sacerdote.

- Es un referenteentemas deformación vinculados a iniciativas pastorales y conocimiento del magisterio de la Iglesia.

El diccionario describe los comportamientos correspondientes a cuatro niveles de apropiación de cada una de las competencias genéricas indispensables. Estos niveles están ordenados de menor a mayor apropiación. El nivel "necesita mejorar" describe comportamientos deficientes, por debajo del desempeño estándar de la competencia. El nivel “estándar" representa los comportamientos suficientes para cumplir con las exigencias propias de la labor del sacerdote diocesano en Perú. Por encima de este nivel se encuentran los niveles "superior" y "muy superior". Los comportamientos de nivel "superior" indican que la persona no sólo ha apropiado suficientemente la competencia, sino que la promueve en su entorno, a partir de la propia vivencia, incluso de manera explícita. Los comportamientos de nivel "muy superior" manifiestan que además la persona es claramente proactiva en la promoción de la competencia y es considerada modélica en su apropiación, por lo que las personas de su entorno tienden a recurrir a ella en busca de orientación.

Cada nivel de apropiación contiene un elenco de entre dos y cuatro comportamientos que lo describen. El número depende en cada caso de que éstos sean suficientes para identificar el correspondiente nivel.

\section{Discusión y Conclusiones}

En relación a la selección de las cinco competencias genéricas indispensables, se constata que los resultados representan diversos aspectos de la vida del sacerdote diocesano: su relación con Dios (Vida interior), sus relaciones con la autoridad jerárquica (Obediencia), sus relaciones interpersonales (Liderar con el ejemplo, Fraternidad sacerdotal), y el propio cultivo personal (Formación permanente).

Llama la atención la ausencia de la competencia Vocación de servicio, que junto con Vida interior fue una de las dos competencias presentes en el discurso de todos los obispos entrevistados para la construcción del perfil que sirvió como base del estudio. No obstante, la dimensión de servicio a los demás está implicada por las competencias, Liderar con el ejemplo, Obediencia y Fraternidad sacerdotal.

Una limitación de la metodología empleada, está en el recurso de una sola persona para la definición de las competencias genéricas indispensables. Por ello, es de gran importancia la elección de la persona adecuada para dicha tarea. Se optó por recurrir al representante de los obispos en Perú. En la organización jerárquica de la Iglesia Católica, la siguiente instancia a la que se podría haber recurrido habría sido una autoridad en la Santa Sede, pero consideramos que con ello se habría perdido la representatividad de la experiencia sacerdotal de un obispo en Perú. 
Se constata como una fortaleza de esta investigación, haber contado con la participación de un porcentaje representativo, de la población de obispos en Perú en la identificación de los comportamientos del diccionario.

El diccionario construido en el segundo estudio aporta, desde la psicología, un conjunto de comportamientos que representan diversos niveles de apropiación de las competencias genéricas indispensables en la labor del sacerdote diocesano en Perú. Proporciona indicadores concretos y mensurables que pueden ser un apoyo en los procesos de formación de los sacerdotes. Complementan las descripciones que desde su propia perspectiva brindan los documentos de la Iglesia Católica.

La pregunta principal de investigación, “¿Cuáles son los comportamientos necesarios para la realización de la labor del sacerdote diocesano en Perú?", se ve respondida por los comportamientos que en el diccionario describen el nivel de apropiación "estándar". El diccionario describe asimismo, en los niveles de apropiación "superior" y "muy superior", comportamientos por encima del "estándar" requerido.

La identificación de las cinco competencias genéricas indispensables, así como el diccionario de comportamientos asociado, son generalizables para la toda la población de sacerdotes diocesanos en Perú.

A partir del trabajo realizado, se sigue la tarea de construir un formato para la evaluación de los niveles de apropiación de las competencias genéricas descritas en los seminaristas y sacerdotes diocesanos en Perú.

Se podría complementar este estudio con la construcción de un diccionario de comportamientos que mida las competencias genéricas que no fueron consideradas indispensables.

\section{REFERENCIAS}

Alles, M. (2009). Nuevo enfoque. Diccionario de comportamientos. La trilogía. Buenos Aires: Granica.

Alonso-Martín, P. (2010). La importancia y el nivel de desarrollo de las competencias en psicología. Psicología Desde El Caribe, 25, 84-107.

Concilio Ecuménico Vaticano II. (1964). Constitución Gaudium et spes. Madrid: Biblioteca de Autores Cristianos.

Catecismo de la Iglesia Católica. (1992). Madrid: Asociación de editores del Catecismo.

Cheney, D. (2013). Current dioceses in Peru. Recuperado de http://www.catholic-hierarchy. org/country/dpe2.html

Código de Derecho Canónico. (1983). Madrid: Biblioteca de Autores Cristianos.

Conferencia Episcopal Peruana. (2013). Mapa de las jurisdicciones eclesiásticas del Perú. Recuperado de http://www.iglesiacatolica.org.pe/cep/map_je_ nondetail.htm

Congregación para la Educación Católica. (2008). Orientaciones para el uso de las competencias de la psicología en la admisión y formación de los candidatos al sacerdocio. Recuperado de http:// www.vatican.va/roman_curia/congregations/ ccatheduc/documents/rc_con_ccatheduc_doc_ 20080628_orientamenti_sp.html

Cortada de Kohan, N., Macbeth, G., López, A. (2008). Técnicas de investigación científica. Buenos Aires: Lugar Editorial.

Escurra, L. (1989) Cuantificación de la Validez de Contenido por Criterio de Jueces. Revista de Psicología - PUCP, 6, 103-111.

Denzin, N. \& Lincoln, Y. Introduction: The Discipline and Practice of Qualitative Research. En Denzin, N. \& Lincoln, Y. (Eds.).(2000). Handbook of Qualitative Research. (pp. 1-28). Thousand Oaks: SAGE.

Finney, D. (2009). The road to self-efficacy: A discussion of generic training in mental health competencies for educational professionals. Pastoral Care In Education, 27(1), 21-28. doi: 10.1080/02643940902731831 
García, J., De la Roche, O., \& Pérez, J. (2007). Ensayo sobre la evaluación de la educación basada en competencia. Revista de la Facultad de Medicina de la UNAM, 50(5), 208-209.

Gvaramadze, I. (2012). Developing generic competences in online virtual education programmes at the University of Deusto. CampusWide Information Systems, 29(1), 4-20. doi: 10.11 08/10650741211192028

Hernández Sampieri, R., Fernández Collado, C., \& Baptista Lucio, M. P. (2006). Metodología de la investigación. Ciudad de México: McGraw-Hill Interamericana.

Kahane, E. (2008). Competency management: Cracking the code. $T+D, 62(5), 71-76$.

Nikolaou, I. (2003). The development and validation of a measure of generic work competencies. International Journal Of Testing, 3(4), 309-319.

Oliva, P. F., \& Henson, K. T. (1980). What are the essential generic teaching competencies? Theory Into Practice, 19(2), 117.
Saracho, J. M. (2005). Un modelo general de gestión por competencias. Santiago de Chile: Ril Editores.

Swinnen, J. (2014). Construcción de un perfil por competencias genéricas para el sacerdote diocesano en Perú. Manuscrito presentado para su publicación.

Strauss, A. y Corbin, J. (2002) Bases de la investigación cualitativa: Técnicas y procedimientos para desarrollar teoría fundamentada. Antioquia: Universidad de Antioquia.

Tobón, S. (2009). Formación basada en competencias. Bogotá: Ecoe.

Torres, E., \& Gutiérrez, I. (2007). Competencias directivas del docente universitario. Revista Panamericana de Pedagogía: Saberes y Quehaceres del Pedagogo, 10, 61-83.

Vasilachis de Gialdino, I. (Ed.). (2006). Estrategias de investigación cualitativa. Barcelona: Gedisa.

Fecha de recepción: 23 de mayo del 2014

Fecha de aceptación: 14 de julio del 20 